evidence that BF duration influences other allergic outcomes, and no evidence that timing of SFI influences any of the outcomes assessed.

Conclusion Longer breastfeeding duration may protect against wheezing later in childhood. Any effect is likely to be through effects on lung function rather than allergic sensitisation. Other allergic outcomes do not appear to be influenced by breastfeeding duration.

\section{P217 CHRONIC MUCUS HYPERSECRETION MAY REPRESENT A BIOMARKER OF AIRWAYS DISEASE ACTIVITY RATHER THAN SIMPLY A PHENOTYPE: A LONGITUDINAL STUDY OF A NATIONALLY REPRESENTATIVE BRITISH BIRTH COHORT}

${ }^{1} \mathrm{JP}$ Allinson, ${ }^{2} \mathrm{R}$ Hardy, ${ }^{1} \mathrm{GC}$ Donaldson, ${ }^{3}$ SO Shaheen, ${ }^{2} \mathrm{D}$ Kuh, ${ }^{1} \mathrm{JA}$ Wedzicha. ${ }^{1}$ National Heart and Lung Institute, Imperial College London, London, UK; ${ }^{2}$ MRC Unit for Lifelong Health and Ageing at UCL, University College London, London, UK; ${ }^{3}$ Centre for Primary Care and Public Health, Barts and the London School of Medicine and Dentistry, London, UK

\subsection{6/thoraxjnl-2015-207770.353}

Introduction Chronic mucus hypersecretion $(\mathrm{CMH})$ is associated with COPD development and progression. $\mathrm{CMH}$ presence across adult life is dynamic, influenced by factors such as smoking behaviour. $\mathrm{CMH}$ is usually considered a binary phenotype and the potential influence of longitudinal $\mathrm{CMH}$ pattern on concurrent $\mathrm{FEV}_{1}$ decline has not been explored. We investigated how longitudinal prevalence of $\mathrm{CMH}$ relates to concurrent $\mathrm{FEV}_{1}$ decline.

Methods The MRC National Survey of Health and Development consists of a sample of men and women born in one week in March 1946 within England, Scotland and Wales. Smoking behaviour, MRC questionnaire defined $\mathrm{CMH}$, height, weight and pre-bronchodilator spirometry were recorded at three ages: 43,53 and (60-64) years.

We used the number occasions that $\mathrm{CMH}$ was positively reported (0-3) as a measure of longitudinal prevalence of $\mathrm{CMH}$. Multilevel models adjusted for sex were used to analyse the relationship between longitudinal prevalence of $\mathrm{CMH}$ and concurrent $\mathrm{FEV}_{1}$ decline (between ages 43 and (60-64)), allowing both intercept and slope to vary according to the longitudinal prevalence of $\mathrm{CMH}$ score. Height, weight and mean $\mathrm{FEV}_{1}$ at age 43 years were then included in the model. Smoking status (current, ex and never-smoker) and number of cigarettes smoked daily were included as time-varying covariates capable of influencing both intercept and slope.

Results 1960 individuals contributed data to the multilevel model: 46\% male; 59\% ever-smoker and mean $\mathrm{FEV}_{1}$ at age 43 years $=3.00$ L. $13 \%$ reported $\mathrm{CMH} \geq$ once between ages 43 and 60-64 years. After full adjustment, longitudinal prevalence of $\mathrm{CMH}$ was significantly associated with both a lower $\mathrm{FEV}_{1}$ at age 43 (intercept $\mathrm{p}<0.001$ ) and a faster decline (slope $\mathrm{p}=$ 0.003) (See Table 1). For each additional occasion $\mathrm{CMH}$ was reported there was an additional $3.2 \mathrm{ml} / \mathrm{yr}$ decline in $\mathrm{FEV}_{1}(\mathrm{p}=$ 0.003 ) i.e. presence of $\mathrm{CMH}$ on all three occasions was associated with an additional $9.6 \mathrm{ml} /$ year $\mathrm{FEV}_{1}$ decline compared with those without $\mathrm{CMH}$ on any occasion.

Conclusion Longitudinal prevalence of $\mathrm{CMH}$ is associated with concurrent $\mathrm{FEV}_{1}$ decline independent of concurrent smoking history. Rather than $\mathrm{CMH}$ being solely an airway disease phenotype, the longitudinal course of $\mathrm{CMH}$ may represent a biomarker of concurrent disease activity.

\section{P218 THE EPIDEMIOLOGICAL, HEALTHCARE AND SOCIETAL BURDEN AND COSTS OF ASTHMA IN THE UK AND MEMBER NATIONS: ANALYSES OF NATIONAL DATABASES}

${ }^{1} \mathrm{M}$ Mukherjee, ${ }^{1} \mathrm{~A}$ Stoddart, ${ }^{2} \mathrm{R}$ Gupta, ${ }^{3} \mathrm{~B}$ Nwaru, ${ }^{4} \mathrm{M}$ Heaven, ${ }^{5} \mathrm{~A}$ Farr, ${ }^{5} \mathrm{D}$ Fitzsimmons, ${ }^{4} \mathrm{~A}$ Bandyopadhyay, ${ }^{6} \mathrm{C}$ Aftab, ${ }^{3} \mathrm{C}$ Simpson, ${ }^{4} \mathrm{R}$ Lyons, ${ }^{7} \mathrm{C}$ Fischbacher, ${ }^{8} \mathrm{C}$ Dibben, ${ }^{9} \mathrm{M}$ Shields, ${ }^{5} \mathrm{C}$ Phillips, ${ }^{2} \mathrm{D}$ Strachan, ${ }^{10} \mathrm{G}$ Davies, ${ }^{1} \mathrm{~B}$ McKinstry, ${ }^{3} \mathrm{~A}$ Sheikh. ${ }^{1}$ Edinburgh Health Services Research Unit, Centre for Population Health Sciences, The University of Edinburgh, Edinburgh, UK; ${ }^{2}$ Population Health Research Institute, St George's, University of London, Cranmer Terrace, London, London, UK; ${ }^{3}$ Asthma UK Centre for Applied Research, Usher Institute of Population Health Sciences and Informatics, The University of Edinburgh, Edinburgh, UK; ${ }^{4}$ Farr Institute, College of Medicine, Swansea University, Singleton Park, Swansea, UK; ${ }^{5}$ Swansea Centre for Health Economics (SCHE), College of Human and Health Science, 2nd Floor Vivian Tower, Swansea University, Singleton Park, Swansea, UK; ${ }^{6}$ The Royal College of Surgeons of Edinburgh \& the University of Edinburgh, Nicolson Street, Edinburgh, UK; ${ }^{7}$ Information Services Division (ISD), NHS National Services Scotland, Room 111, Gyle Square, 1 South Gyle Crescent, Edinburgh, UK; ${ }^{8}$ School of Geography \& Geosciences, Department of Geography \& Sustainable Development, The University of Edinburgh, Edinburgh, UK; ${ }^{9}$ Centre for Infection and Immunity, School of Medicine, Dentistry and Biomedical Sciences, Health Sciences Building, Queen's University Belfast, 97 Lisburn Road, Belfast, UK; ${ }^{10}$ Asthma \& Allergy Group, Institute of Life Science, Swansea University Medical School, Singleton Park, Swansea, UK

10.1136/thoraxjnl-2015-207770.354

Abstract P217 Table 1 The association between longitudinal prevalence of CMH (number of occasions $\mathrm{CMH}$ reported) and FEV $\mathrm{V}_{1}$ between ages 43 and 60-64 years. Multilevel model includes 1960 individuals

\begin{tabular}{|c|c|c|c|c|c|}
\hline & & \multicolumn{2}{|c|}{$\begin{array}{l}\text { FEV }_{1} \text { intercept (mls) } \\
\text { at age } 43 \text { years }\end{array}$} & \multicolumn{2}{|c|}{$\begin{array}{l}\text { FEV }_{1} \text { linear change per year ( } \mathrm{mls} / \text { year) } \\
\text { between ages } 43 \text { and }(60-64) \text { years }\end{array}$} \\
\hline & & Coefficient ( $95 \% \mathrm{Cl}$ ) & pvalue & Coefficient $(95 \% \mathrm{Cl})$ & pvalue \\
\hline \multirow{2}{*}{$\begin{array}{c}\text { Per occasion CMH } \\
\text { reported between ages } \\
43 \text { and (60-64) years } \\
\text { (0-3 occasions) }\end{array}$} & Minimally adjustedt & -90.0 (-46.8 to -133.3$)$ & $0 \quad 0.001$ & $-10.2(-12.8$ to -7.7$)$ & $<0.001$ \\
\hline & Fully adjusted $\neq$ & -143.4 ( -104.1 to -182.8$)$ & $0<0.001$ & $-3.2(-1.1$ to -5.3$)$ & 0.003 \\
\hline
\end{tabular}

† Adjusted for sex and age

₹ Adjusted for sex, age, height at age 43 years, weight at age 43 years, smoking status and smoking intensity at ages 43,53 and (60-64) years 
Background Developing a comprehensive picture of the burden of asthma in the UK will enable informed national decisions about care provision and planning. We sought to provide the first UK-wide estimates of the epidemiology, healthcare utilisation and costs of asthma.

Methods We undertook analyses of national health surveys, routine healthcare and administrative datasets over the period 2010-12. Economic modelling was carried out to estimate costs. Estimates were calculated for each nation and the UK as a whole.

Results The UK lifetime prevalence of patient-reported symptoms suggestive of asthma in 2010-11 was 30.7\% (95\% Confidence Intervals [CI] 29.2-32.2; equivalent to [ ] 18,949,516 people), lifetime prevalence of patient-reported physician-diagnosed asthma was $15.9 \%$ (95\% CI 14.7-17.1; 10,841,030 people), annual prevalence of patient-reported physician-diagnosed-and-treated asthma was 9.1\% $\quad(95 \%$ CI $\quad 8.0-10.2$; $\sim 5,765,237$ people), annual prevalence of GP reported-anddiagnosed asthma was $8.2 \%$ (95\% CI 8.2-8.2; 5,215,607 people) and annual prevalence of GP reported-and-diagnosed-andtreated asthma was 6.0\% (95\% CI 6.0-6.0; 3,946,796 people). In 2011-12, asthma resulted in an estimated: 6,392,670 primary-care consultations; 93,916 inpatient-care episodes; 1,864 (317 paediatric and 1,547 adult) intensive-care unit episodes; 36,800 disability living allowance (DLA) claims; and 1,160 deaths. The estimated cost of asthma in the UK was at least $£ 1.1$ billion in $2011-12$ : $75 \%$ of this was for primary-care $(60 \%$ prescribing and $15 \%$ consultations), $13 \%$ for DLA claims, and $10 \%$ for hospital care.

Conclusions We found that asthma is very common, affecting at least 3.95 million people, and that it is responsible for substantial morbidity, healthcare and societal costs in the UK. Setting ambitious targets for improving asthma outcomes is paramount and resources should be targeted to improving community-based prescribing decisions and reducing the risk of asthma exacerbations and associated hospitalisations and deaths.

Funding Asthma UK, with additional support from the Edinburgh Health Services Research Unit and Farr Institute, UK.

\section{P219 POTENTIAL IMPACT OF AIR POLLUTION COVERAGE IN THE MEDIA ON RESPIRATORY DISEASE ADMISSIONS}

${ }^{1}$ PS Tang, ${ }^{2} \mathrm{D}$ Shaw, ${ }^{3} \mathrm{G}$ Smith, ${ }^{3} \mathrm{~J}$ Goulding. ${ }^{1}$ East Midlands Academic Health Sciences Network; ${ }^{2}$ Division of Respiratory Medicine, University of Nottingham, Nottingham, UK; ${ }^{3}$ Horizon Digital Economy Research, University of Nottingham, Nottingham, UK

\subsection{6/thoraxjnl-2015-207770.355}

Introduction Air pollution can exacerbate respiratory disease. However, when air quality warnings are provided by authoritative bodies (e.g. MET office) media coverage may be disproportionate. We explored whether there is an association between respiratory admissions and media pollution coverage via non-linear predictive models, to potentially predict respiratory admissions.

Methods The association was examined as follows:

1. Baseline regression models were generated to predict daily respiratory admission episodes from $1^{\text {st }}$ January 2009-9 $9^{\text {th }}$ April 2014. Predictors consisted of daily logs for PM10 particulate matter, PM2.5, Nitric oxide, Nitrogen dioxide, Ozone, Black carbon, Mean Temperature, Precipitation (obtained from National Oceanic and Atmospheric Administration data) and the DAQI Air Pollution Index. Models were optimised via cross-validation using daily respiratory admission levels sourced from Nottingham University Hospitals Trust data (ICD10 codes J39 - J9999).

2. Time series of levels of media coverage were generated by applying kernel density estimation at a range of bandwidths (using linear and exponential kernels at bandwidths of 1, 10, 25,50 and 100 days) to daily counts of online news articles featuring pollution and air quality issues over the period 01.01.2013 - 9.04. 2014.

3. Predictive model accuracies were compared following integration of these time series of media coverage levels as an additional predictor.

Results Of the predictive models tested, random forests parameterized provided optimal results for air-quality predictors. When predicting daily respiratory admissions, the model's accuracy was $19.90 \%$ better than simply predicting mean daily admissions, with an average root mean square error (RMSE) of 7.5031. However, on introduction of the media-coverage variable, RMSE was reduced to 7.3210 , representing a $21.85 \%$ improvement over mean prediction. While this reflected a slight improvement in admissions forecasting, a corrected t-test suggested these differences were not statistically significant, with a p-value of 0.0633 .

Conclusion Initial results indicate that consideration of media coverage may offer minor improvements in predicting respiratory admissions, but this effect was not statistically significant. While such a relationship requires further investigation, models informed by media coverage cannot currently be considered to be accurate enough for use in a practical setting. Better media data collection may improve prediction accuracy.

\section{P220 USING FUNNEL PLOTS TO MAKE MEANINGFUL CENTRE COMPARISONS}

${ }^{1} \mathrm{~L}$ Pierotti, ${ }^{2} \mathrm{MA}$ Mohammed, ${ }^{3} \mathrm{M}$ Wildman, ${ }^{4} \mathrm{D}$ Bilton, ${ }^{5} \mathrm{~J}$ Boote, ${ }^{4} \mathrm{SB}$ Carr, ${ }^{6} \mathrm{~K}$ Collins, ${ }^{1} \mathrm{P}$ Cullinan, ${ }^{7} \mathrm{C}$ Elston, ${ }^{3} \mathrm{~S}$ Harrison, ${ }^{8} \mathrm{P}$ Norman, ${ }^{9} \mathrm{SJ}$ MacNeill. ${ }^{1}$ Imperial College London, London, UK; ${ }^{2}$ University of Bradford, Bradford, UK; ${ }^{3}$ Sheffield Teaching Hospitals, Sheffield, UK; ${ }^{4}$ Royal Brompton Hospital NHS Trust, London, UK; ${ }^{5}$ University of Hertfordshire, Hatfield, UK; ${ }^{6}$ National Services Scotland, Edinburgh, UK; ${ }^{7}$ King's College Hospital NHS Trust, London, UK; ${ }^{8}$ University of Leeds, Leeds, UK; ${ }^{9}$ University of Bristol, Bristol, UK

\subsection{6/thoraxjnl-2015-207770.356}

Objectives The UK CF Registry annual reports include comparisons between centres on key outcomes such as FEV1 using rankings. While illustrating the distribution between centres, they promote the assumption that those with the highest measures provide "better" care. We hypothesised a more scientific approach based on statistical "process control" using funnel plots and adjustment for case-mix may help to identify exceptional CF care services in terms of clinically meaningful outcomes.

Methods We extracted data from annual reviews (2007-2012) on the CF Registry. Our outcomes included FEV1 (\% predicted) at 15 years and change in FEV1 between 18 and 21 years. Funnel plots were generated with confidence limits at 2 and 3 standard deviations (SD). Centres with mean values outside these limits are said to display "special cause variation" -variability outside what one would expect. Outcomes were then adjusted for case mix (including gender, genotype, pancreatic sufficiency and socio-economic deprivation) and analysed using funnel plots.

Results 31 paediatric centres provided FEV1 data on 15 year olds between 2007 and 2012. Funnel plots of unadjusted FEV1 (\% predicted) showed few centres with evidence of special cause variation (2SD limits). Initial case-mix adjustment reduced the number of centres outside these limits to 3 . We also identified 28 adult centres providing sufficient data to calculate change in 